Supporting Information for

\title{
Tumbling of Anisole Units in Calixarene Promotes Its Shuttling in Rotaxanes
}

Haochuan Chen, ${ }^{\dagger, \mathrm{O}}$ Hong Zhang, ${ }^{\dagger, \mathrm{O}}$ Xueguang Shao, ${ }^{\dagger,+,},\left\|_{\text {and Wensheng Cai }}{ }^{*},,\right\|$

${ }^{\dagger}$ Research Center for Analytical Sciences, College of Chemistry, Nankai University,

Tianjin Key Laboratory of Biosensing and Molecular Recognition, Tianjin 300071, China.

${ }^{\ddagger}$ State Key Laboratory of Medicinal Chemical Biology, Tianjin 300071, China

"Collaborative Innovation Center of Chemical Science and Engineering, Tianjin, 300071, China

*Corresponding E-mail: wscai@ nankai.edu.cn (W. C.) 


\section{Collective variables in free-energy calculation}

Detailed definition of $i$. The collective variable $i$ is used to describe the inversion process of the calix[6]arene wheel. To define $i$, the central plane across the six anisole units of the wheel is determined by least squares approximation first. That is to say, let the equation of the central plane be $k_{0} x+k_{1} y+z+k_{2}=0$ and suppose the factor of $z$ is not zero since the central plane is impossible parallel to the $\mathrm{z}$ axis. We get following equations by using the least squares algorithm to fit the plane along the center-of-mass of each phenyl rings at the calix[6]arene:

$$
\left\{\begin{array}{c}
\tilde{z}_{j}=-k_{0} x_{j}-k_{1} y_{j}-k_{2} \\
\frac{\partial}{\partial k_{0}} \sum_{j}\left(\tilde{z}_{j}-z_{j}\right)^{2}=0 \\
\frac{\partial}{\partial k_{1}} \sum_{j}\left(\tilde{z}_{j}-z_{j}\right)^{2}=0 \\
\frac{\partial}{\partial k_{2}} \sum_{j}\left(\tilde{z}_{j}-z_{j}\right)^{2}=0
\end{array}\right.
$$

The $\left(x_{j}, y_{j}, z_{j}\right)$ denotes the Cartesian coordinate of the center-of-mass of the $j$-th phenyl ring. Solving eq. 1 and the factor $k_{0}, k_{1}$ and $k_{2}$ can be computed as

$$
\left\{\begin{array}{c}
k_{0}=\frac{\sum x_{j} z_{j}\left(\left(\sum y_{j}\right)^{2}-n \sum y_{j}{ }^{2}\right)+\sum y_{j} z_{j}\left(n \sum x_{j} y_{j}-\sum x_{j} \sum y_{j}\right)+\sum z_{j}\left(\sum x_{j} \sum y_{j}{ }^{2}-\sum y_{j} \sum x_{j} y_{j}\right)}{\sum x_{j}{ }^{2}\left(n \sum y_{j}{ }^{2}-\left(\sum y_{j}\right)^{2}\right)-\sum x_{j} y_{j}\left(n \sum x_{j} y_{j}-\sum x_{j} \sum y_{j}\right)+\sum x_{j}\left(\sum x_{j} y_{j} \sum y_{j}-\sum x_{j} \sum y_{j}{ }^{2}\right)} \\
k_{1}=\frac{\sum x_{j} z_{j}\left(n \sum x_{j} y_{j}-\sum x_{j} \sum y_{j}\right)+\sum y_{j} z_{j}\left(\left(\sum x_{j}\right)^{2}-n \sum x_{j}{ }^{2}\right)+\sum z_{j}\left(\sum x_{j}{ }^{2} \sum y_{j}-\sum x_{j} \sum x_{j} y_{j}\right)}{\sum x_{j}{ }^{2}\left(n \sum y_{j}{ }^{2}-\left(\sum y_{j}\right)^{2}\right)-\sum x_{j} y_{j}\left(n \sum x_{j} y_{j}-\sum x_{j} \sum y_{j}\right)+\sum x_{j}\left(\sum x_{j} y_{j} \sum y_{j}-\sum x_{j} \sum y_{j}{ }^{2}\right)} \\
k_{2}=\frac{\sum x_{j} z_{j}\left(\sum y_{j}{ }^{2} \sum x_{j}-\sum y_{j} \sum x_{j} y_{j}\right)+\sum y_{j} z_{j}\left(\sum x_{j}{ }^{2} \sum y_{j}-\sum x_{j} \sum x_{j} y_{j}\right)+\sum z_{j}\left(\left(\sum x_{j} y_{j}\right)^{2}-\sum x_{j}{ }^{2} y_{j}{ }^{2}\right)}{\sum x_{j}{ }^{2}\left(n \sum y_{j}{ }^{2}-\left(\sum y_{j}\right)^{2}\right)-\sum x_{j} y_{j}\left(n \sum x_{j} y_{j}-\sum x_{j} \sum y_{j}\right)+\sum x_{j}\left(\sum x_{j} y_{j} \sum y_{j}-\sum x_{j} \sum y_{j}{ }^{2}\right)}
\end{array}\right.
$$

where the $n$ is the total number of phenyl rings. The normal vector of the central plane, $\mathbf{o}$, can then be expressed as

$$
\mathbf{o}=\left(\frac{k_{0}}{\sqrt{k_{0}{ }^{2}+k_{1}{ }^{2}+1}}, \frac{k_{1}}{\sqrt{k_{0}{ }^{2}+k_{1}{ }^{2}+1}}, \frac{1}{\sqrt{k_{0}{ }^{2}+k_{1}{ }^{2}+1}}\right)
$$

After defining the central plane, the projection of the $j$-th phenyl ring along the normal vector $\mathbf{o}, p_{j}$ is computed as $p_{j}=\mathbf{r}_{j} \cdot \mathbf{o}$, where the vector $\mathbf{r}$ connects the first 
and fourth atoms of the $j$-th phenyl ring. Finally, the collective variable $i$ can be obtained as

$$
i=\sum_{j=1}^{6} p_{j}
$$

Restricted by the rigidity of the phenyl ring, the length of $\mathbf{r}$ can be approximated at $2.8 \AA$, and as a result, $p_{j}$ ranges from -2.8 to $2.8 \AA$ and $i$ ranges from -16.8 to $16.8 \AA$.

Using the collective variable $i$ in simulations. To improve the reproducibility of the present work and ease the burden on using the collective variable $i$, a source-code patch

of NAMD 2.13b1, ${ }^{1}$ which enables the computing of $i$ in the colvars module, ${ }^{2}$ is provided as fit_plane.patch in SI.zip. The users can download the NAMD 2.13b1 and apply using "patch -Np1 -i fit_plane.patch" assuming source code of NAMD and the patch are in the same working directory. Additional "make depends" command is required before typing "make" to compile the NAMD source.

\section{Representative structures}

Figure $\mathrm{S} 1$ shows the least free-energy pathway $\mathrm{P}_{1}$, where the movements of inversion and translation are coupled, and another probable pathway $\mathrm{P}_{2}$, where the two movements are decoupled. Figures S2-S5 depict the representative structures along $\mathrm{P}_{1}$, and Figure S6, $\mathrm{S} 7$ and $\mathrm{S} 5$ show the representative structures along $\mathrm{P}_{2}$. 


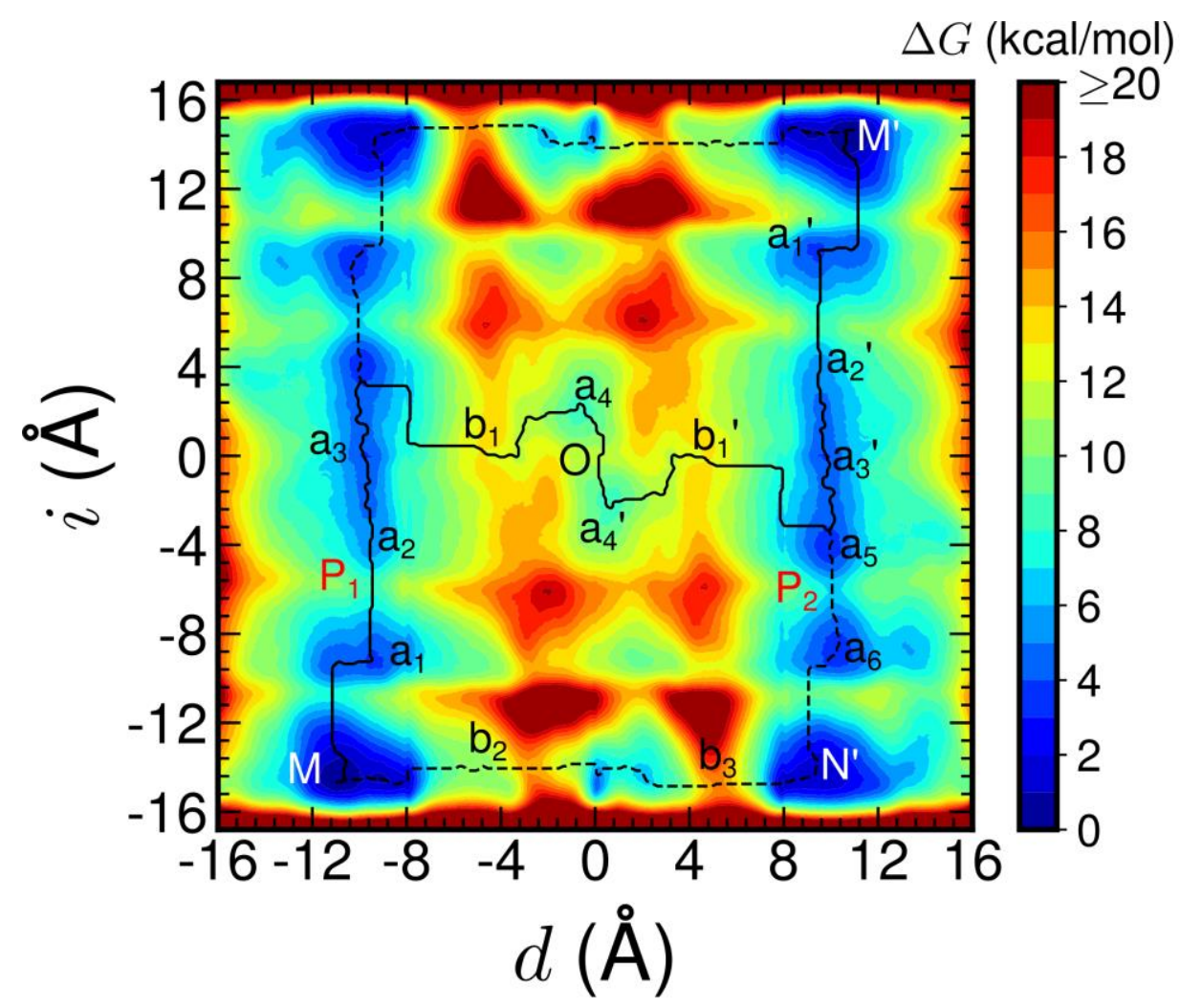

Figure $\mathrm{S} 1$. The free-energy pathways of $\mathrm{P}_{1}$ and $\mathrm{P}_{2} . \mathrm{P}_{1}$ goes via $\mathrm{M}, \mathrm{a}_{1}, \mathrm{a}_{2}, \mathrm{a}_{3}, \mathrm{~b}_{1}, \mathrm{a}_{4}, \mathrm{O}, \mathrm{a}_{4}$, $b_{1}{ }^{\prime}, a_{3}{ }^{\prime}, a_{2}{ }^{\prime}, a_{1}{ }^{\prime}$ and $M^{\prime}$. $P_{2}$ goes via $M, b_{2}, b_{3}, N^{\prime}, a_{6}, a_{5}, a_{3}{ }^{\prime}, a_{2}{ }^{\prime}, a_{1}$ ' and $M^{\prime}$. The number of tumbled anisole units of the macrocycle at different regions is listed as following: $\mathrm{M}$, $b_{2}, b_{3}$ and N': no tumbled anisole unit; $a_{1}$ and $a_{6}:$ one tumbled anisole units; $a_{2}$ and $a_{5}$ : two tumbled anisole units; $\mathrm{a}_{3}, \mathrm{~b}_{1}, \mathrm{O}, \mathrm{b}_{1}$ ' and $\mathrm{a}_{3}$ ': three tumbled anisole units, $\mathrm{b}_{1}$ and $\mathrm{b}_{1}$ ' denote the free-energy barriers along $\mathrm{P}_{1}$; $\mathrm{a}_{4}$ : two anisole units are tumbled while one unit is tumbling; $\mathrm{a}_{4}$ ': three anisole units are tumbled while one unit is tumbling; $\mathrm{a}_{2}{ }^{\prime}$ : four anisole units are tumbled; $\mathrm{a}_{1}$ ': five anisole units are tumbled; M': six anisole units are tumbled. 


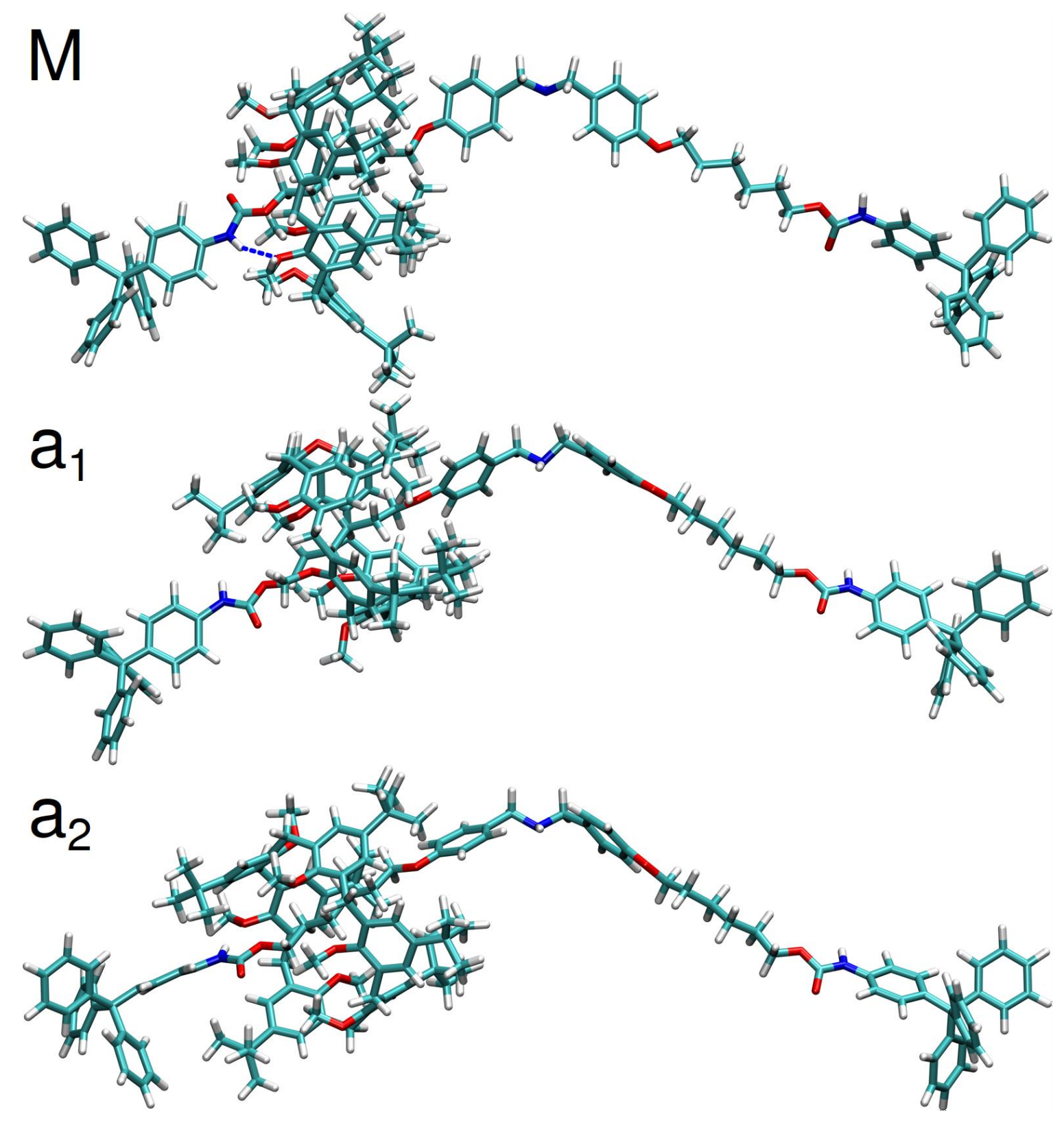

Figure S2. Representative structures of $\mathrm{M}, \mathrm{a}_{1}$ and $\mathrm{a}_{2}$. Conformations of the calix[6]arene macrocycle: $M$ : cone; $\mathrm{a}_{1}: 1$-alternate; $\mathrm{a}_{2}: 1,3$-alternate. 

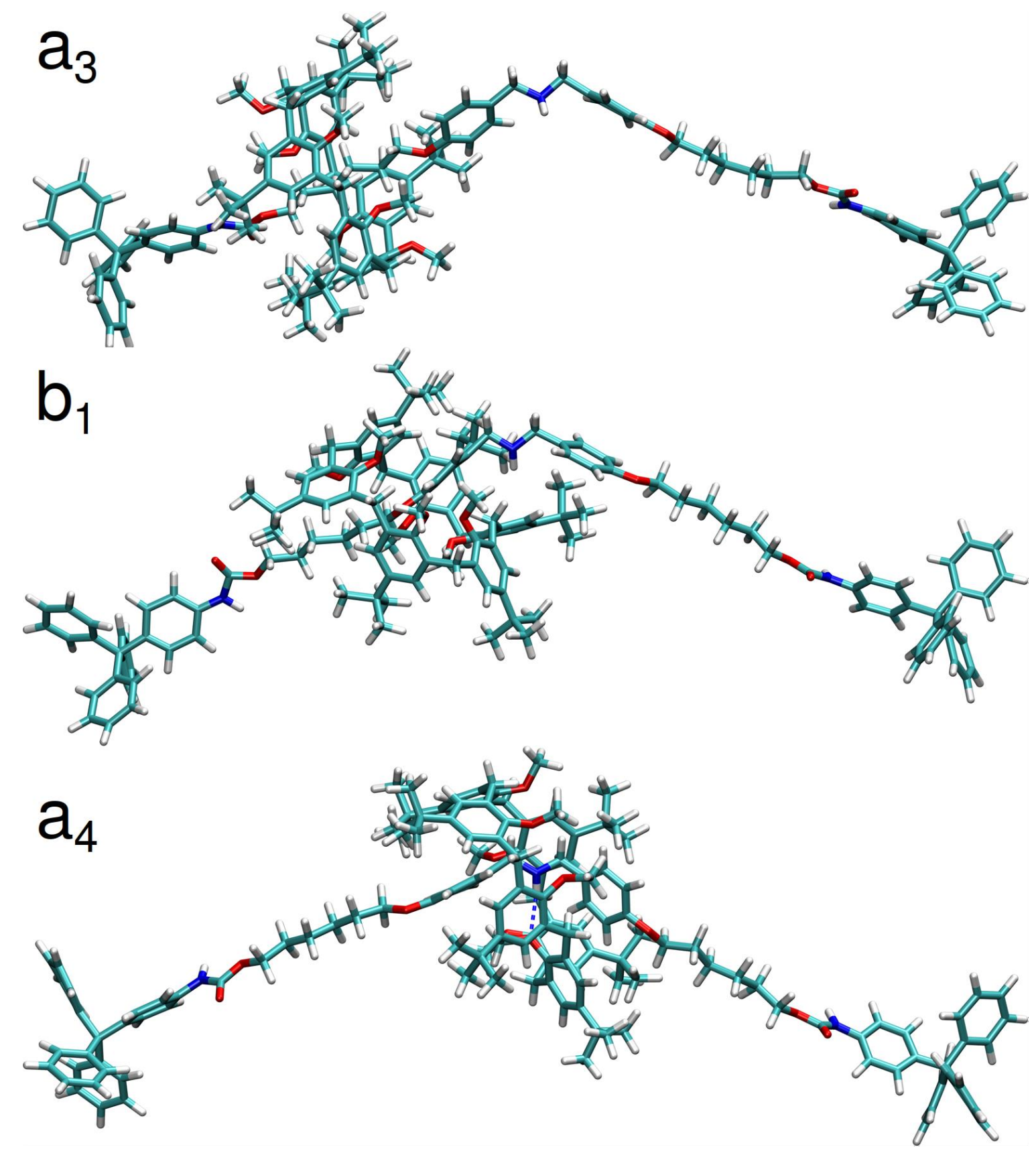

Figure S3. Representative structures of $a_{3}, b_{1}$ and $a_{4}$. Conformations of the calix[6]arene macrocycle: $a_{3}:$ 1,2,3-alternate; $b_{1}: 1,2,3$-alternate; $a_{4}$ : partial 1,2,3-alternate. 


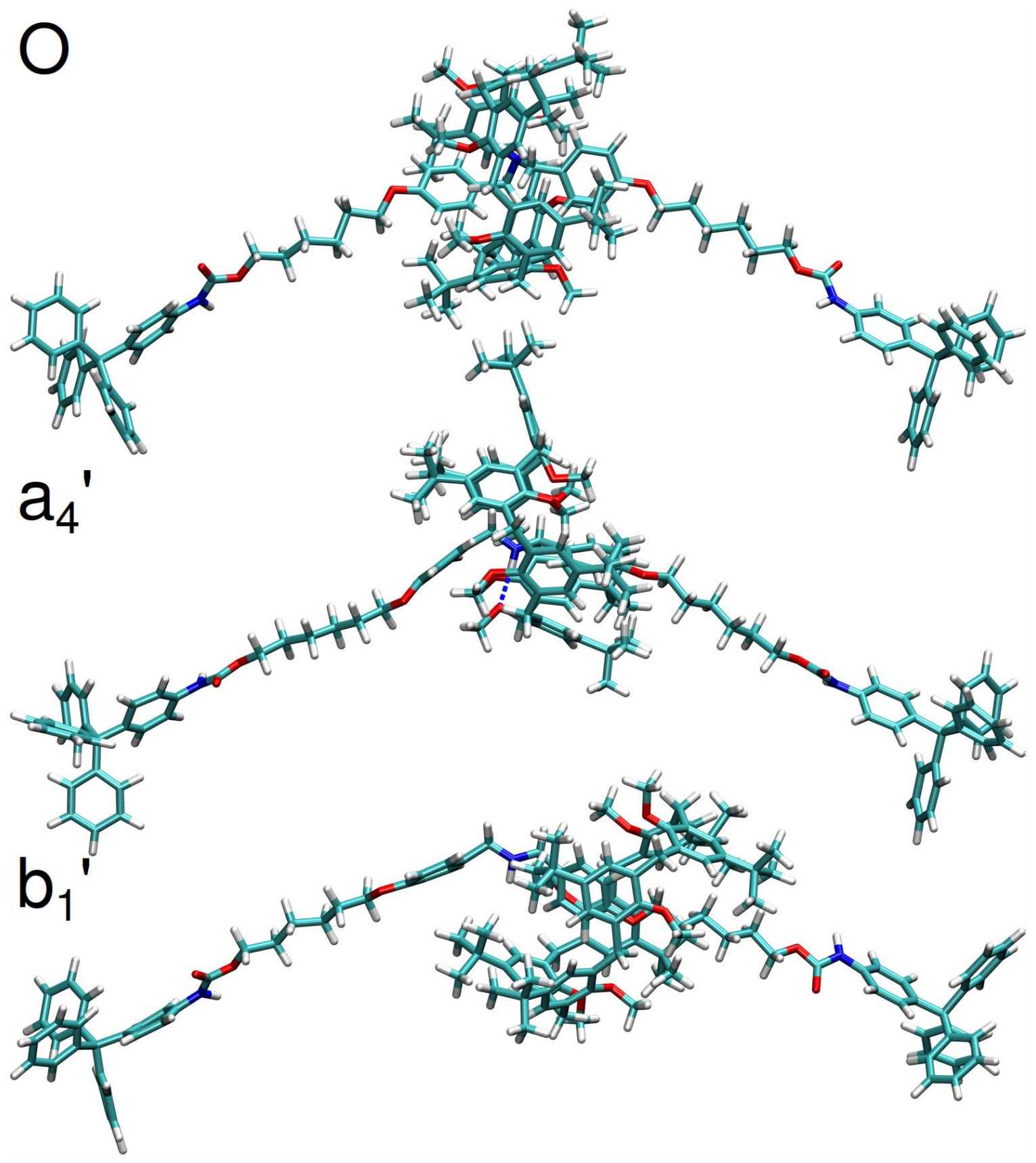

Figure S4. Representative structures of $\mathrm{O}, \mathrm{a}_{4}$ ' and $\mathrm{b}_{1}$ '. Conformations of the calix[6]arene macrocycle: O: 1,2,3-alternate; $a_{4}$ ': partial 1,2,3-alternate; $b_{1}{ }^{\prime}: 1,2,3$-alternate. 

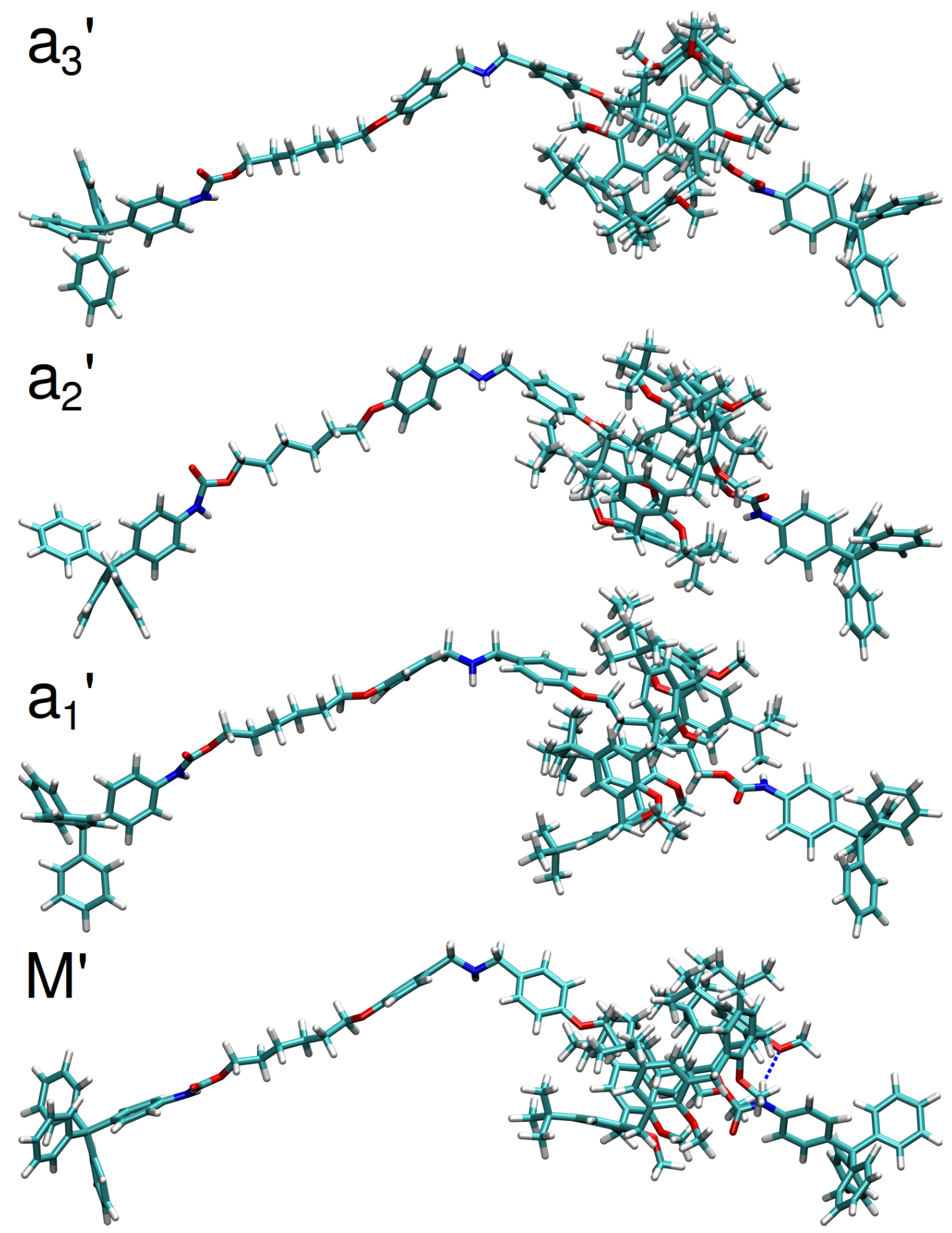

Figure S5. Representative structures of $a_{3}{ }^{\prime}, a_{2}$ ', $a_{1}$ ' and M'. Conformations of the calix[6]arene macrocycle: $a_{3}{ }^{\prime}:$ 1,2,3-alternate; $a_{2}{ }^{\prime}:$ 1,2,3,5-alternate (inverted 
1,3-alternate); $\mathrm{a}_{1}{ }^{\prime}: 1,2,3,4,5$-alternate (inverted 1-alternate); $\mathrm{M}^{\prime}$ : inverted cone.

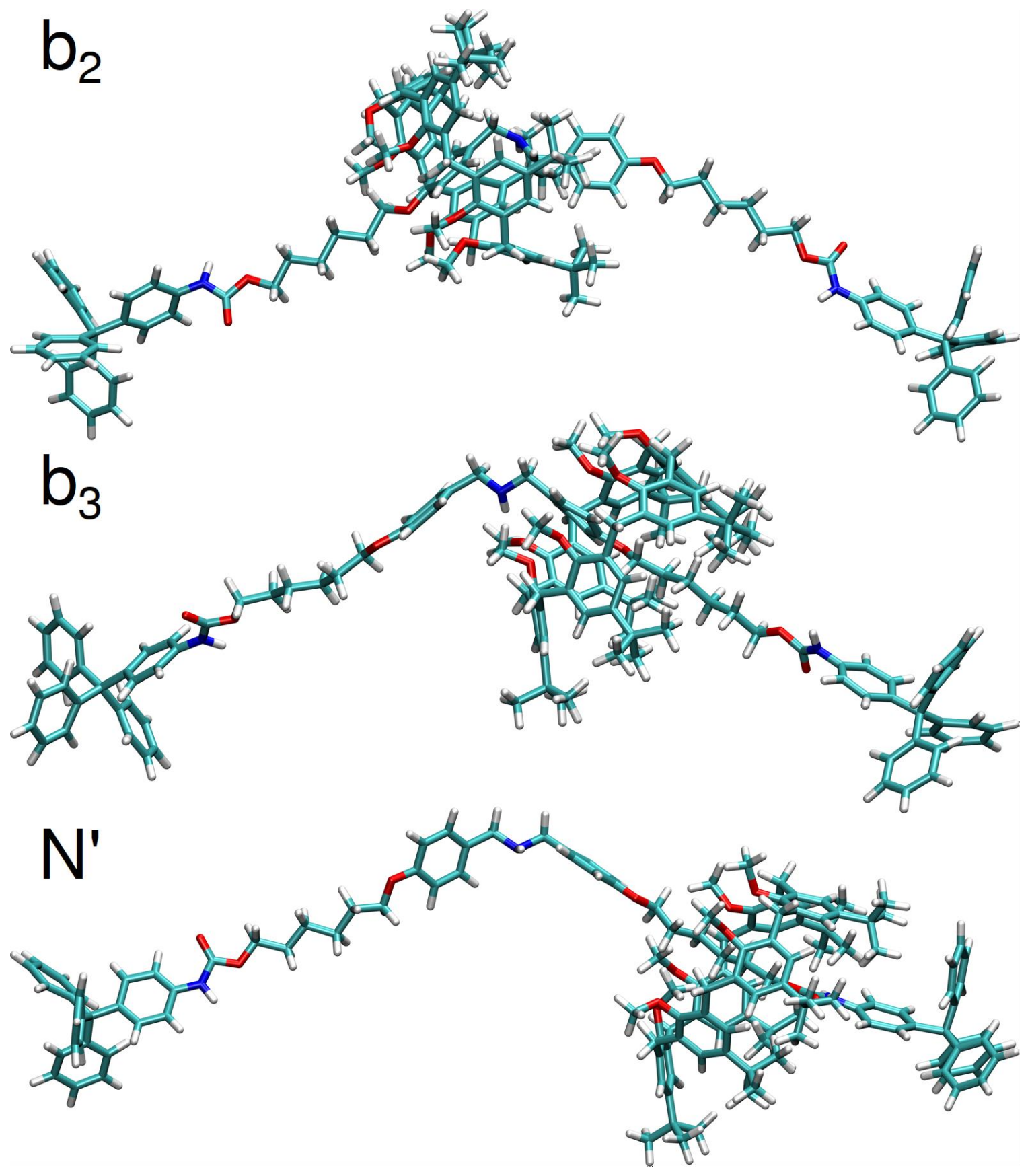

Figure S6. Representative structures of $b_{2}, b_{3}$ and N'. Conformations of the calix[6]arene macrocycle: $b_{2}, b_{3}$ and $N^{\prime}$ : cone. 


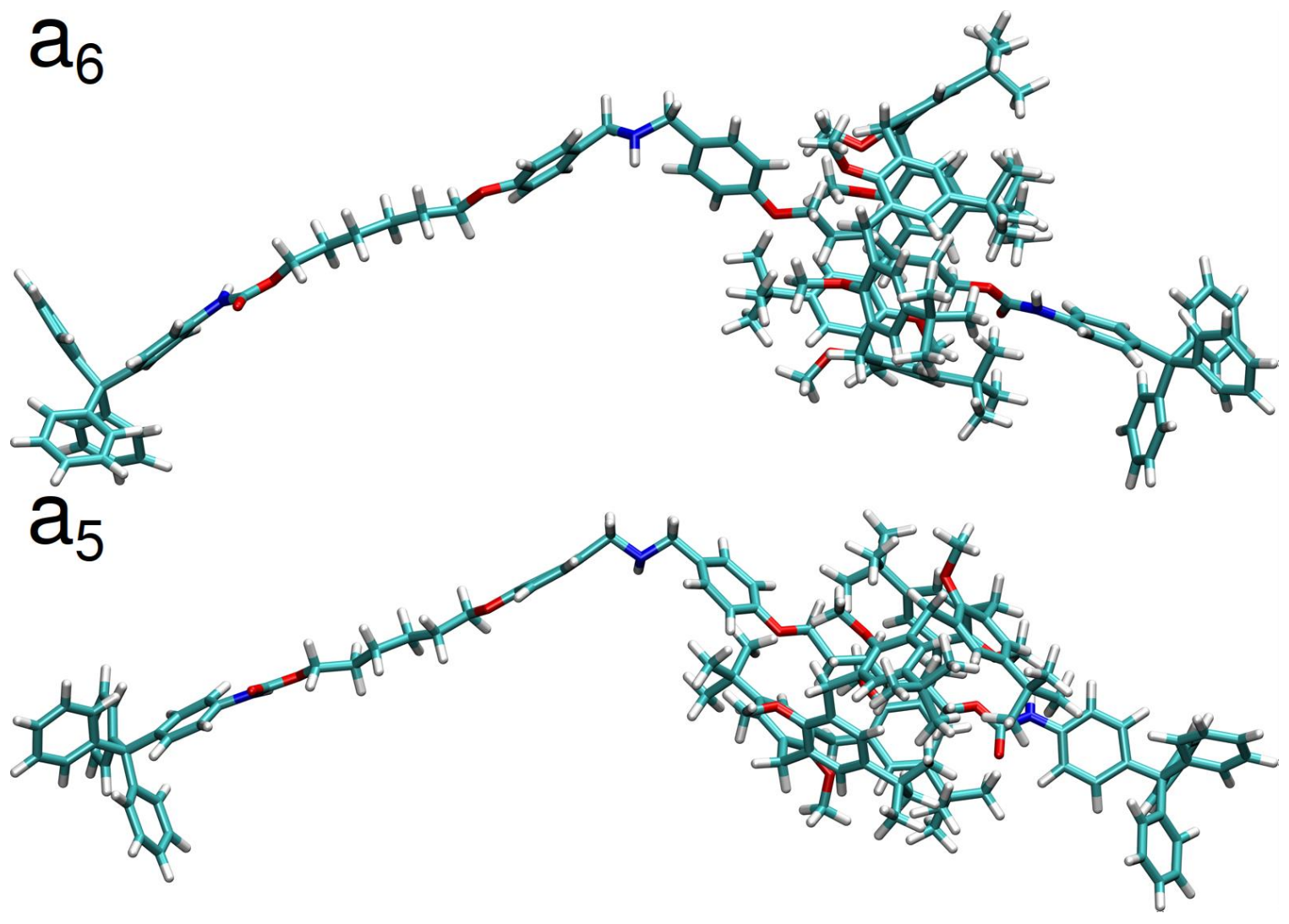

Figure S7. Representative structures of $\mathrm{a}_{6}$ and $\mathrm{a}_{5}$. Conformations of the calix[6]arene macrocycle: $\mathrm{a}_{6}$ : 1-alternate; $\mathrm{a}_{5}: 1,3$-alternate.

\section{Statistical analysis of tumbled anisole units}


Table S1 Relation between the the number of tumbled anisole units, the conformation of calix[6]arene macrocycle and the approximated value of $S$

\begin{tabular}{|c|c|c|}
\hline $\begin{array}{l}\text { Number of } \\
\text { tumbled units }\end{array}$ & Conformation & $S$ (Approximated) \\
\hline 0 & $0($ cone $)$ & 0 \\
\hline \multirow[t]{2}{*}{1} & 1(1-alternate) & $1,2,4,8,16,32$ \\
\hline & 2a(1,2-alternate $)$ & $3,6,12,24,33,48$ \\
\hline \multirow[t]{3}{*}{2} & $2 \mathrm{~b}(1,3$-alternate $)$ & $5,10,17,20,34,40$ \\
\hline & $2 c(1,4$-alternate $)$ & $9,18,36$ \\
\hline & $3 \mathrm{a}(1,2,3$-alternate $)$ & $7,14,28,35,56,49$ \\
\hline \multirow[t]{3}{*}{3} & $3 b(1,2,4$-alternate $)$ & $11,13,19,22,25,26,37,38,41,44,50,52$ \\
\hline & $3 c(1,3,5$-alternate $)$ & 21,42 \\
\hline & 4a(1,2,3,4-alternate $)$ & $15,30,39,51,57,60$ \\
\hline \multirow[t]{2}{*}{4} & $4 \mathrm{~b}(1,2,3,5$-alternate $)$ & $23,29,43,46,53,58$ \\
\hline & $4 \mathrm{c}(1,2,4,5$-alternate $)$ & $27,45,54$ \\
\hline 5 & 5(inverted 1-alternate) & $31,47,55,59,61,62$ \\
\hline 6 & 6(inverted cone) & 63 \\
\hline
\end{tabular}

${ }^{\mathrm{a}} \mathrm{A}$ schematic representation of the conformation of $2 \mathrm{a}, 2 \mathrm{~b}, 2 \mathrm{c}, 3 \mathrm{a}, 3 \mathrm{~b}, 3 \mathrm{c}, 4 \mathrm{a}, 4 \mathrm{~b}$ and $4 \mathrm{c}$ can be found at Figure 4A.

Statistical results of the calix[6]arene model threaded by octadecane chain. The statistical results of the module where the calix[6]arene macrocycle is threaded by an octadecane chain are shown in Table S2. 
Table S2 Statistical results of the octadecane threaded calix[6]arene model

\begin{tabular}{|c|c|c|c|c|c|c|c|c|c|c|c|c|c|}
\hline \multirow{3}{*}{ minimum } & \multicolumn{13}{|c|}{ Ratio of conformations (\%) } \\
\hline & \multirow{2}{*}{0} & \multirow{2}{*}{1} & \multicolumn{3}{|c|}{2} & \multicolumn{3}{|c|}{3} & \multicolumn{3}{|c|}{4} & \multirow{2}{*}{5} & \multirow{2}{*}{6} \\
\hline & & & $2 \mathrm{a}$ & $2 b$ & $2 \mathrm{c}$ & $3 a$ & $3 b$ & $3 \mathrm{c}$ & $4 a$ & $4 \mathrm{~b}$ & $4 \mathrm{c}$ & & \\
\hline $\mathrm{T}_{0}$ & 100 & 0 & & 0 & & & 0 & & & 0 & & 0 & 0 \\
\hline $\mathrm{T}_{1}$ & 0 & 100 & & 0 & & & 0 & & & 0 & & 0 & 0 \\
\hline $\mathrm{T}_{2}$ & 0 & 0 & 0 & 99.54 & 0.39 & & 0.06 & & & 0 & & 0 & 0 \\
\hline $\mathrm{T}_{3}$ & 0 & 0 & & 0.04 & & 9.28 & 9.57 & 81.09 & & 0 & & 0 & 0 \\
\hline $\mathrm{T}_{2}{ }^{\prime}$ & 0 & 0 & & 0 & & & 0.48 & & 11.00 & 87.80 & 0.66 & 0.06 & 0 \\
\hline $\mathrm{T}_{1}{ }^{\prime}$ & 0 & 0 & & 0 & & & 0 & & & 0.16 & & 99.78 & 0.05 \\
\hline $\mathrm{T}_{0}{ }^{\prime}$ & 0 & 0 & & 0 & & & 0 & & & 0 & & 0 & 100 \\
\hline
\end{tabular}

${ }^{\mathrm{a}} \mathrm{A}$ schematic representation of the conformation of $2 \mathrm{a}, 2 \mathrm{~b}, 2 \mathrm{c}, 3 \mathrm{a}, 3 \mathrm{~b}, 3 \mathrm{c}, 4 \mathrm{a}, 4 \mathrm{~b}$ and $4 \mathrm{c}$ can be found at Figure 4A.

\section{Shortest cavity diameter of the calix[6]arene macrocycle}

Methods and results. Since the calix[6]arene is a symmetrical macrocycle and has even number of anisole units, the minimum distances of the opposite anisole units, $d_{i, j}^{\min }$, can be used to access the shortest cavity diameter (SCD) of the calix[6]arene backbone. Precisely speaking, assuming the six anisole units are indexed from one to six, there are three pairs of minimum distances, namely $d_{1,4}^{\min }, d_{2,5}^{\min }$ and $d_{3,6}^{\min }$, which are computed as the distance between the two closest atoms at the first and fourth, second and fifth and 
third and sixth anisole units. The final measurement of narrowness, $d_{\text {min }}$, is defined as the minimum value of the three pairs of minimum distance, namely

$d_{\min }=\min \left(d_{1,4}^{\min }, d_{2,5}^{\min }, d_{3,6}^{\min }\right)$. Figure S8 shows the SCD results at the free-energy

barriers of the paths $\mathrm{P}_{1}$ and $\mathrm{P}_{2}$.
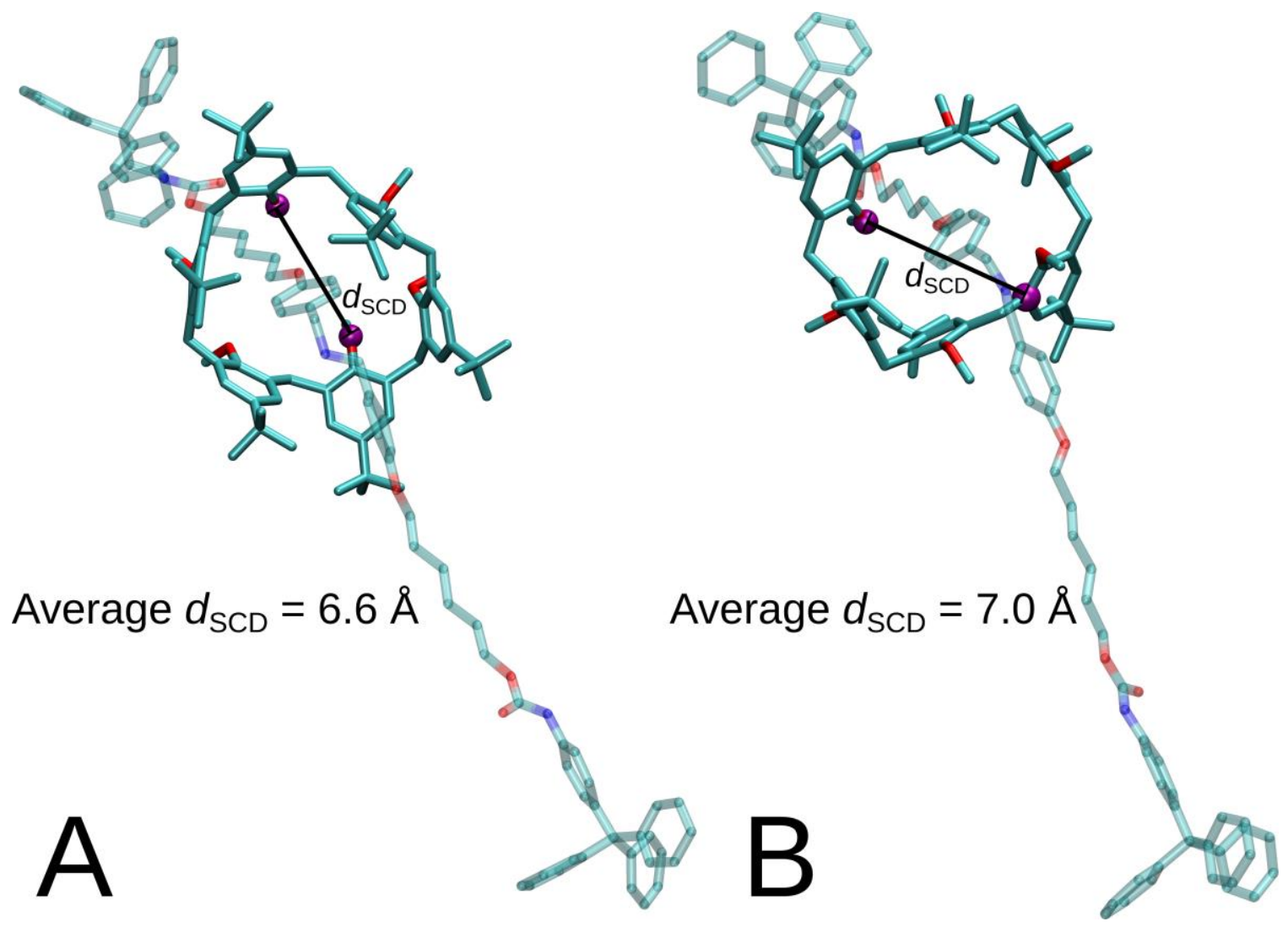

Figure S8. (A) SCD and representative structure at the free-energy barrier of $\mathrm{P}_{2}$. (B) SCD and representative structure at the free-energy barrier of $P_{1}$.

Statistical results of the calix[6]arene threaded by the deprontonated dialkylammonium chain. The ratio of different conformations of the calix[6]arene with deprontonated dialkylammonium chain is evaluated and presented as a simplified pie chart in Figure 4A. The detailed version is shown in Figure S9 below. 


\section{Conformations and ratio(\%)}

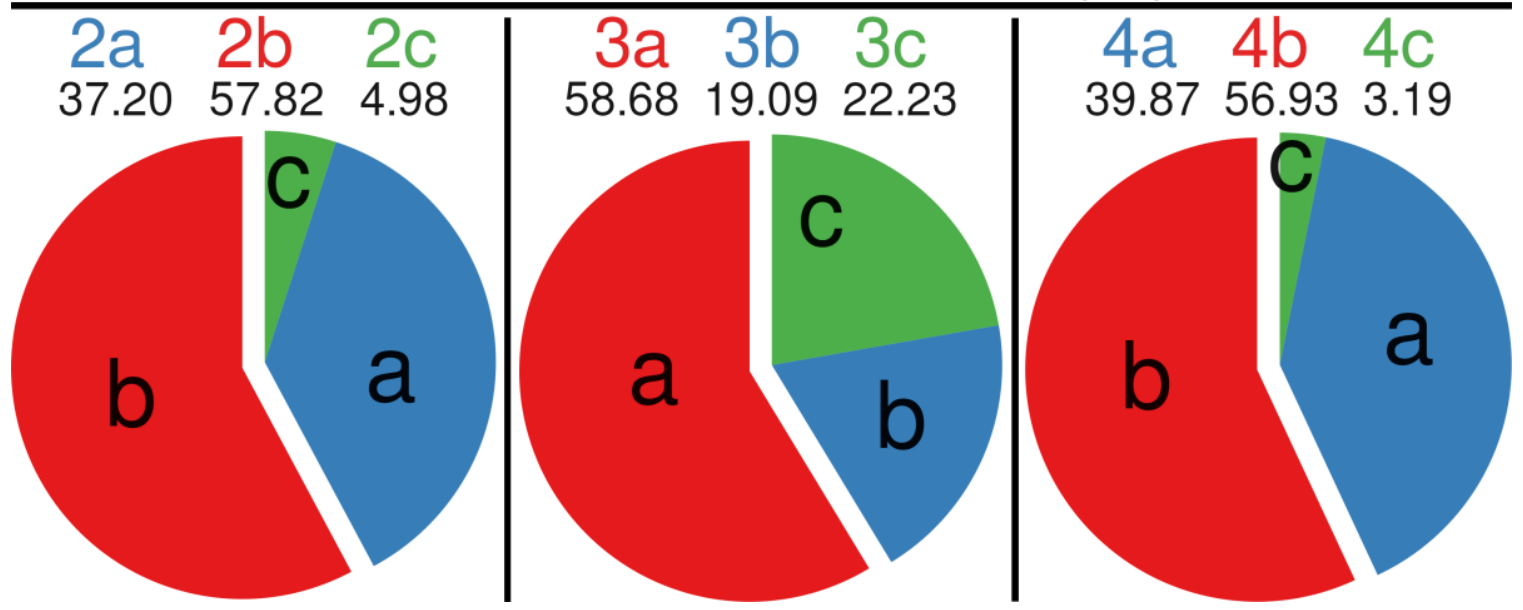

Figure S9. Ratio of different conformations of the calix[6]arene when there are 2, 3 or 4 tumbled anisole units.

\section{Electrostatic potential map}

The electrostatic potential maps of the calix[6]arene and the chains studied in this work are presented in Figures S10-S12.

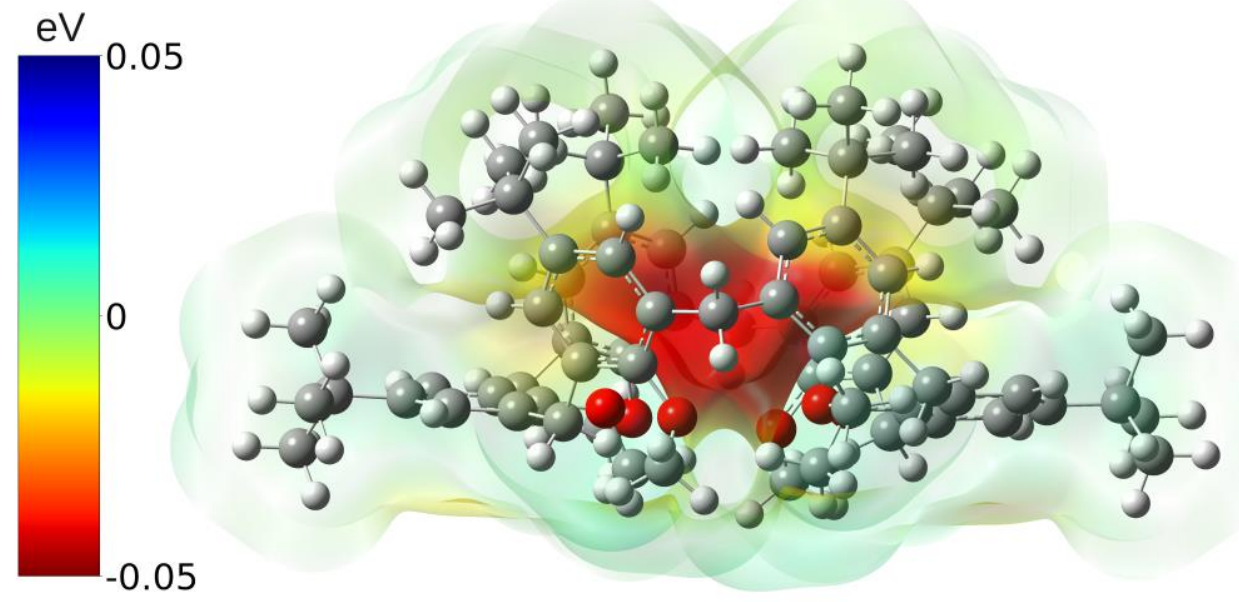

Figure S10. Electrostatic potential map of the calix[6]arene in cone conformation. 


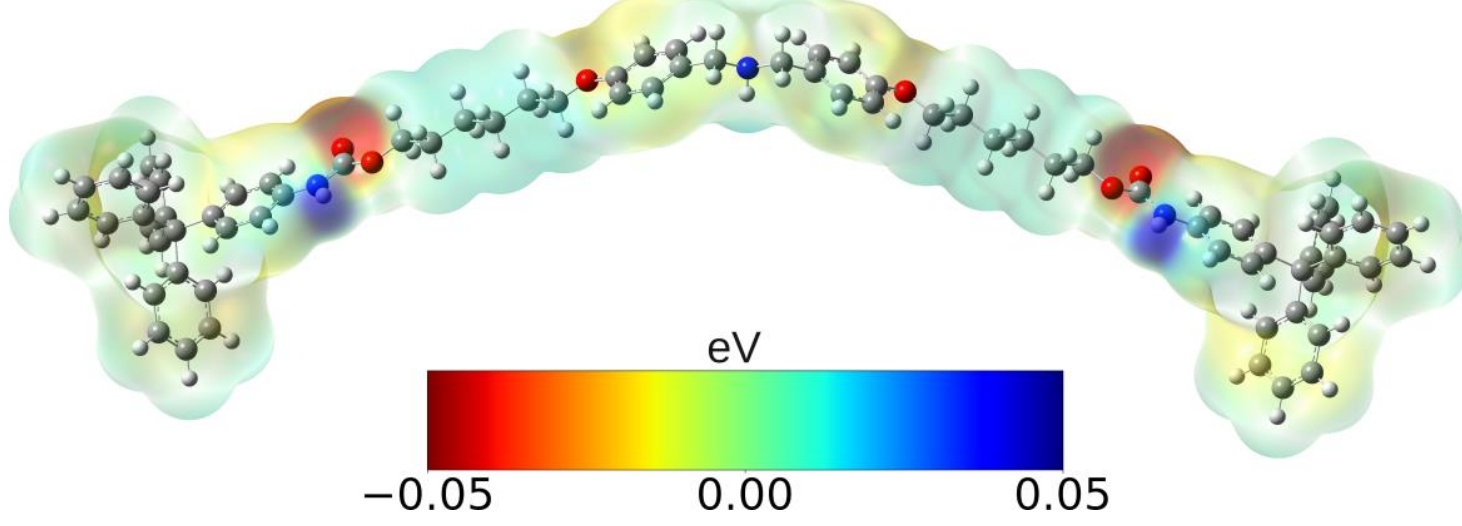

Figure S11. Electrostatic potential map of the chain in the rotaxane studied shown in Scheme 1.

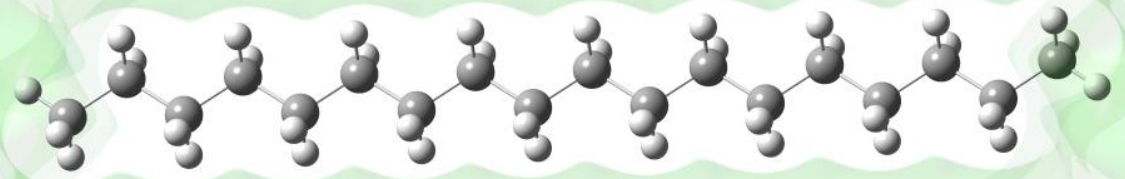

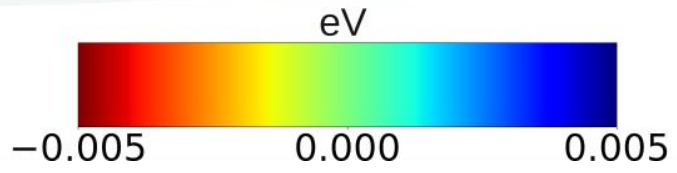

Figure S12. Electrostatic potential map of the octadecane chain in the test model shown in Figure 2.

\section{References}

(1) Phillips, J. C.; Braun, R.; Wang, Wei.; Gumbart, J.; Tajkhorshid, E.; Villa, E.; Chipot, C.; Skeel, R. D.; Kalé, L.; Schulten, K. Scalable Molecular Dynamics with NAMD. J. Comput. Chem. 2005, 26, 1781-1802. 
(2) Fiorin, G.; Klein, M. L.; Hénin, J. Using Collective Variables to Drive Molecular Dynamics Simulations. Mol. Phys. 2013, 111, 3345-3362. 